\title{
Macroscopic Electron Quantum Coherence in a Solid-State Circuit
}

\author{
H. Duprez, ${ }^{1, *}$ E. Sivre, ${ }^{1, *}$ A. Anthore, ${ }^{1,2}$ A. Aassime, ${ }^{1}$ A. Cavanna, ${ }^{1}$ A. Ouerghi, ${ }^{1}$ U. Gennser, ${ }^{1}$ and F. Pierre ${ }^{1, \dagger}$ \\ ${ }^{1}$ Centre de Nanosciences et de Nanotechnologies (C2N), CNRS, Univ Paris Sud, \\ Université Paris-Saclay, 91120 Palaiseau, France \\ ${ }^{2}$ Université de Paris, Univ Paris Diderot, 75013 Paris, France
}

(Received 18 December 2018; published 14 May 2019)

\begin{abstract}
The quantum coherence of electronic quasiparticles underpins many of the emerging transport properties of conductors at small scales. Novel electronic implementations of quantum optics devices are now available with perspectives such as "flying-qubit" manipulations. However, electronic quantum interferences in conductors remained up to now limited to propagation paths shorter than $30 \mu \mathrm{m}$ independent of the material. Here we demonstrate strong electronic quantum interferences after a propagation along two 0.1 -mm-long pathways in a circuit. Interferences of visibility as high as $80 \%$ and $40 \%$ are observed on electronic analogues of the Mach-Zehnder interferometer of, respectively, 24- $\mu \mathrm{m}$ and 0.1-mm arm length, consistently corresponding to a 0.25 -mm electronic phase coherence length. While such devices perform best in the integer quantum Hall regime at filling factor 2, the electronic interferences are restricted by the Coulomb interaction between copropagating edge channels. We overcome this limitation by closing the inner channel in micron-scale loops of frozen internal degrees of freedom combined with a loop-closing strategy providing an essential isolation from the environment.
\end{abstract}

DOI: 10.1103/PhysRevX.9.021030

Subject Areas: Condensed Matter Physics, Mesoscopics, Quantum Physics

\section{INTRODUCTION}

Ballistic electrons allow for advanced quantum manipulations at the single-electron level in circuits, in the spirit of the manipulation of photons in quantum optics [1-3]. Perspectives notably include a different paradigm for quantum-information processing with a nonlocal architecture based on "flying-qubits" encoded, for example, by the presence or absence of an electron within a propagating wave packet $[1,2,4-7]$. Electronic edge states topologically protected against disorder constitute promising solid-state platforms. In particular, the emblematic chiral edge channels propagating along a two-dimensional (2D) conductor in the quantum Hall regime are generally considered ideal 1D conductors. Their analogy with light beams, their in situ tunability by field effect, and the availability of single-electron emitters were exploited to implement the electronic analogues of optical devices, such as the interferometers of types Fabry-Perot [8], Mach-Zehnder [9], Hanbury-Brown and Twiss [10], and Hong-Ou-Mandel [11]. In contrast to photons, the Coulomb interaction

\footnotetext{
*These authors contributed equally to this work.

frederic.pierre@c2n.upsaclay.fr
}

Published by the American Physical Society under the terms of the Creative Commons Attribution 4.0 International license. Further distribution of this work must maintain attribution to the author(s) and the published article's title, journal citation, and DOI. between charged electrons provides a natural correlation mechanism to realize, e.g., CNOT gates $[1,2,4,5]$. However, the same Coulomb interaction generally entangles the propagating electrons efficiently with numerous degrees of freedom, including the surrounding electrons, which gives rise to quantum decoherence [12] (see Ref. [13] for a notable exception).

In practice, the maximum electron phase coherence length $L_{\phi}$ was previously found to reach remarkably similar values at the lowest accessible temperatures in very diverse systems, from diffusive metal $\left(L_{\phi} \simeq 20 \mu \mathrm{m}\right.$ reported in Ref. [14] at $40 \mathrm{mK}$ ) to near ballistic two-dimensional electron gas ( $L_{\phi} \simeq 20 \mu \mathrm{m}$ reported in Ref. [15] at $30 \mathrm{mK}$ ) and graphene $\left(L_{\phi} \simeq 3-5 \mu \mathrm{m}\right.$ estimated in Ref. [16] at $260 \mathrm{mK}$ ). Along the ballistic quantum Hall edge channels of specific interest for electron quantum optics, $L_{\phi} \simeq$ $24 \mu \mathrm{m}$ was demonstrated at $20 \mathrm{mK}$ [17] at the most advantageous magnetic field tuning corresponding to filling factor $\nu=2$ in a $\mathrm{Ga}(\mathrm{Al}) \mathrm{As} 2 \mathrm{D}$ electron gas. We also point out two promising findings: An important temperature robustness of small conductance oscillations measured across a $6-\mu \mathrm{m}$-long $\mathrm{Ga}(\mathrm{Al}) \mathrm{As}$ device, from which a large value of $L_{\phi} \sim 86 \mu \mathrm{m}$ was indirectly inferred [18] and conductance oscillations of very high visibility along a graphene $p$ - $n$ junction [19]. Here, we establish a macroscopic electron phase coherence length of $0.25 \mathrm{~mm}$ achieved along quantum Hall channels by nanocircuit engineering. 
At low temperatures, short-range electron-electron interactions within the same chiral edge channel of the integer quantum Hall regime are predicted to increase the electrons' propagation velocity but not to limit their coherence [20,21]. The dominant dephasing mechanism is generally attributed to the interaction between electrons located in adjacent edge channels [20,22] (except at $\nu=1$ and fractional filling factors where the stronger decoherence $[23,24]$ is not clearly understood). This picture is established by complementary signatures including energy transfers [25,26], charge fractionalization [27-29], and Hong-Ou-Mandel characterizations [30]. However, additional dissipative mechanisms yet unidentified were also evidenced experimentally, even in the most canonical $\nu=2$ case [25-27]. In this work, we demonstrate a circuit design strategy that very efficiently suppresses the essential decoherence mechanisms.

\section{NANOENGINEERING THE PHASE COHERENCE LENGTH}

The electronic version of the Mach-Zehnder interferometer [MZI, schematically depicted in Fig. 1(a)] essentially consists in a quantum Hall edge channel following two separate paths, and in two quantum point contacts (QPCs) used as tunable beam splitters [9]. The quantum Hall regime is realized in a $\mathrm{Ga}(\mathrm{Al}) \mathrm{As} 2 \mathrm{D}$ electron gas immersed in a perpendicular magnetic field of $4.3 \mathrm{~T}$ corresponding to a filling factor $\nu=2$, with two copropagating edge channels. The interfering MZI paths involve only the outer edge channel [thick black lines in Fig. 1(a)]. The two beamsplitter QPCs are formed by field effect using split gates [colored orange in Fig. 1(a) with suspended bridges to contact the top parts]. The quantum phase difference between the two paths is proportional to the enclosed magnetic flux. It is here controlled by fine-tuning the lower edge path with the voltage $V_{\mathrm{pl}}$ applied to a lateral plunger gate [colored green in Figs. 1(a) and 1(b)]. The quantum interferences are evidenced by sweeping $V_{\mathrm{pl}}$ from the resulting oscillations of the transmitted current impinging on the metallic electrode labeled $D$ in Fig. 1(a). Their energy dependence with respect to the bias voltage $V_{\mathrm{dc}}$ applied to the source electrode is obtained from a concomitant noise in the transmitted current. The second MZI output is connected to the central metallic electrode (elongated yellow disk in Fig. 1), which is electrically grounded through a suspended bridge. In contrast to previous MZI implementations, our devices include two long surface gates [light gray in Figs. 1(a) and 1(b)] with a particular comb shape with both shafts and teeth placed over the 2D electron gas. This shape is essential for the presently demonstrated strong increase of the electron coherence. As illustrated in Fig. 1(a), these gates can be biased to form inner channel loops along the interfering outer edge channel paths. In order to unambiguously demonstrate and accurately measure very large phase

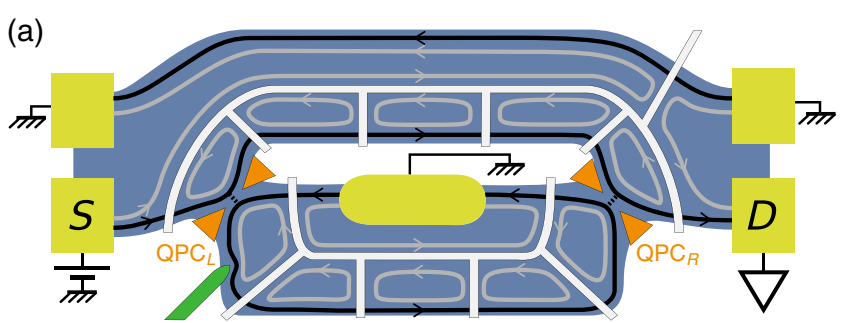

(b)

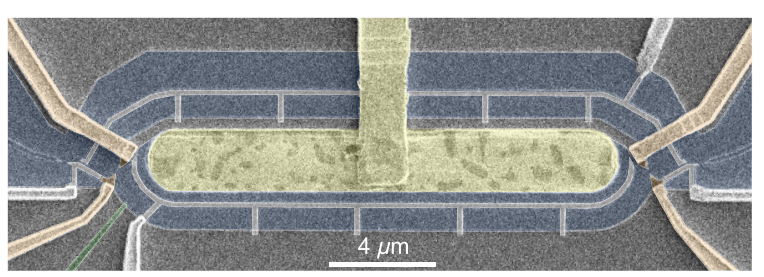

(c)

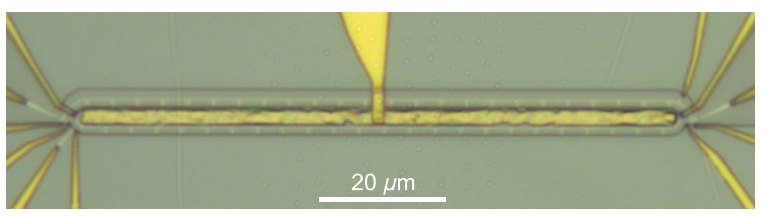

FIG. 1. Nanocircuit engineering of electronic coherence. (a) Sample schematic. Two chiral edge channels (black and gray lines with arrows) propagate along a 2D electron gas (blue) set in the integer quantum Hall regime at filling factor $\nu=2$. The outer channel (black) follows two separate paths between tunable beam splitters implemented by quantum point contacts (orange), thereby forming a Mach-Zehnder interferometer. The inner edge channel (gray) can be closed into well-separated loops with specific comb-shaped gates (light gray) voltage biased to reflect only this channel. Sweeping the voltage on a lateral plunger gate (green) results in MZI oscillations of the current transmitted from source $(S)$ to detector $(D)$. (b) Colored scanning electron micrograph of the sample with MZI arms of symmetric length $L \simeq 24 \mu \mathrm{m}$. (c) Optical image of the $L \simeq 0.1 \mathrm{~mm}$ MZI. The inner edge channel loops have nominally identical perimeters of $9 \mu \mathrm{m}$, except one of $5 \mu \mathrm{m}$ for the lower left loop of each sample.

coherence lengths, we fabricate two MZIs with extraordinarily long symmetric arms of length $L \simeq 24 \mu \mathrm{m}$ [Fig. 1(b)] and $0.1 \mathrm{~mm}$ [Fig. 1(c)]. For a straightforward comparison at different $L$, the two devices are made concurrently (a few millimeters away on the same chip) with identical designs except for the length of the elongated central area and are simultaneously cooled down to $10 \mathrm{mK}$.

How can $L_{\phi}$ be increased? It was initially shown that most of the electrons' energy relaxation can be frozen within the outer edge channel at $\nu=2$ (along an $8-\mu \mathrm{m}$ path) by closing into a loop the inner channel [31]. This freezing was explained by the electronic levels' quantization within the loop, which effectively quenches the phase space for inelastic collisions with the inner loop's electrons (for a level spacing larger than the available energy) [31,32]. As inelastic collisions also result in decoherence, a similar approach was subsequently tested on $L_{\phi}$ using an electronic MZI [33]. However, the increase in $L_{\phi}$ by forming inner channel loops was limited to a factor of 2 [33], relatively 

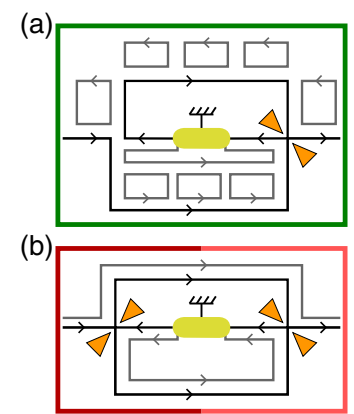

(c)

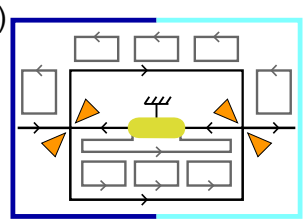

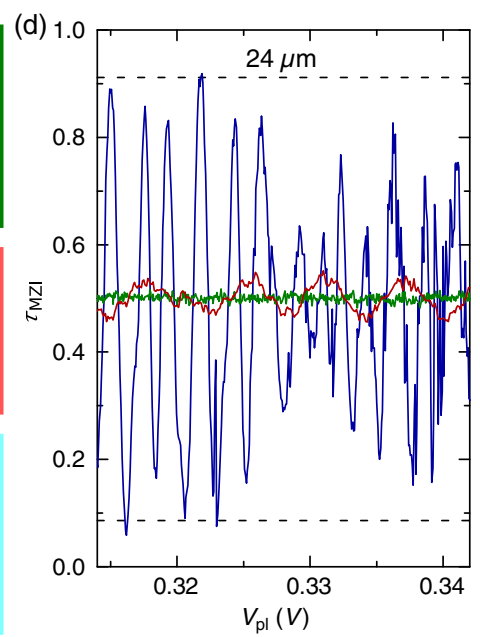
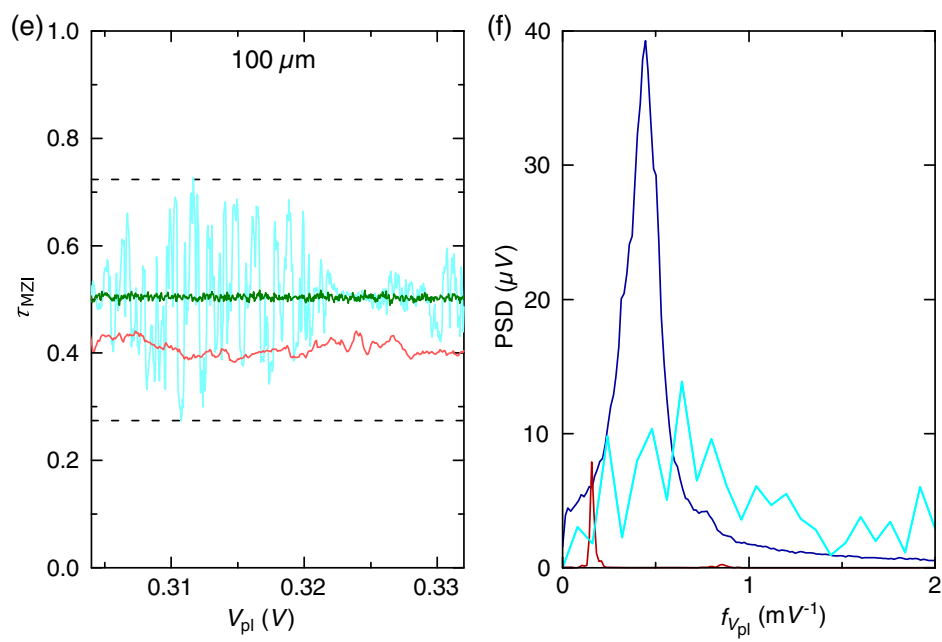

FIG. 2. Quantum oscillations. (a)-(c) Schematics of the different configurations. (d),(e) Continuous lines show versus plunger gate voltage $V_{\mathrm{pl}}$, the measured fraction $\tau_{\mathrm{MZI}}$ of current transmitted from $S$ to $D$ along the outer channel of the $L \simeq 24 \mu \mathrm{m}$ (d) and $0.1 \mathrm{~mm}$ (e) MZI [same color as the box enclosing the corresponding schematic in panels (a), (b), or (c); darker shade for the shorter device]. Horizontal dashed lines display the predicted $\tau_{\text {MZI }}$ extrema for the same $L_{\phi}=0.25 \mathrm{~mm}$ in both MZIs. (f) Continuous lines show the power spectral density of $\tau_{\mathrm{MZI}}\left(V_{\mathrm{pl}}\right)$ determined along large $V_{\mathrm{pl}}$ sweeps (extending between 50 and $80 \mathrm{mV}$ ) measured several times [same color code as in panels (d),(e)]. For the challenging case of $L \simeq 0.1 \mathrm{~mm}$ in configuration (c) (light blue line), the Fourier analysis is restricted to plunger gate-voltage windows exhibiting oscillations larger than $66 \%$ of their maximum amplitude.

modest compared to the freezing of energy relaxation. Our conjecture is that the weaker impact on $L_{\phi}$ reflects a fundamental design limitation in the MZI implementation of Ref. [33], where an otherwise negligible coupling between two different outer edge channels could be mediated by the rigid displacements of the inner loops. These rigid displacements provide an additional mechanism for both decoherence and energy relaxation: Even if the inner loops' electronic degrees of freedom are not excited, the loops' presence can strongly enhance the capacitive coupling between different propagative edge channels adjacent to separate portions of the same loops. The present MZI implementation suppresses this mechanism while preserving a $2 \mathrm{D}$ bulk at $\nu=2$ through a gate design allowing for a much larger separation of the inner loops from additional quantum Hall channels (see Fig. 4 for an illustration, and Sec. II in the Appendix for further discussion).

\section{QUANTUM OSCILLATIONS VERSUS LOOP FORMATION}

We present in Fig. 2 illustrative MZI oscillations versus plunger gate voltage $V_{\mathrm{pl}}$ (a positive bias of $+0.35 \mathrm{~V}$ is applied during cooldown). The displayed $\tau_{\mathrm{MZI}}$ corresponds to the transmission probability across the MZI from source $S$ to detector $D$. It is given by the fraction measured at the electrode $D$ of the current injected into the outer edge channel at the electrode $S$. The two $L \simeq 24 \mu \mathrm{m}$ and $0.1 \mathrm{~mm}$ MZIs are each tuned in three different configurations [Figs. 2(a)-2(c)]. The green lines in Figs. 2(d) and 2(e) are data obtained with both devices set in the configuration shown in Fig. 2(a). Their flatness demonstrates directly in the presence of inner channel loops the absence of $\tau_{\mathrm{MZI}}$ oscillations when all the transmitted current goes through a single MZI arm (the lower arm; in this specific case $\tau_{\mathrm{MZI}}=\tau_{\mathrm{QPC}}^{R}$ since $\left.\tau_{\mathrm{QPC}}^{L}=1\right)$. The red and blue lines in Figs. 2(d) and 2(e) are obtained with both QPC beam splitters set to half transmission probability for the outer edge channel $\left(\tau_{\mathrm{QPC}}^{L} \simeq \tau_{\mathrm{QPC}}^{R} \simeq 0.5\right.$, the inner edge channel being always fully reflected at the QPCs) in the configurations illustrated in Figs. 2(b) and 2(c). In the conventional MZI configuration [no loops, Fig. 2(b)], small oscillations of period $6.4 \mathrm{mV}$ are observed only on the $L \simeq 24 \mu \mathrm{m}$ device [dark red lines in Figs. 2(d) and 2(f)]. Their visibility $\mathcal{V} \equiv\left(\tau_{\mathrm{MZI}}^{\max }-\tau_{\mathrm{MZI}}^{\min }\right) /\left(\tau_{\mathrm{MZI}}^{\max }+\tau_{\mathrm{MZI}}^{\min }\right) \approx 6 \%$ corresponds to a typical phase coherence length value of $L_{\phi} \simeq 17 \mu \mathrm{m}$ (despite a relatively low temperature $T \simeq 10 \mathrm{mK}$ ) obtained from the standard relationship for a symmetric MZI:

$\mathcal{V}=4 \sqrt{\tau_{\mathrm{QPC}}^{R}\left(1-\tau_{\mathrm{QPC}}^{R}\right) \tau_{\mathrm{QPC}}^{L}\left(1-\tau_{\mathrm{QPC}}^{L}\right)} \exp \left(\frac{-2 L}{L_{\phi}}\right)$,

which assumes a perfect absorption of the outer edge channel by the central metallic contact connected to electrical ground (separately checked; see the Appendix). In contrast, for the $L \simeq 0.1 \mathrm{~mm}$ device, no oscillations can be detected without inner channel loops as expected from Eq. (1) $\left(\mathcal{V} \approx 10^{-5}\right.$ calculated with $L=$ $0.1 \mathrm{~mm}$ and $L_{\phi}=17 \mu \mathrm{m}$ ). Instead, we observe a slowly evolving $\tau_{\mathrm{MZI}}$, which is markedly below 0.5 . This low mean value reflects the tunneling of electrons from outer to inner edge channels, which becomes significant over 
such a long propagation distance. As a result, a larger (smaller) fraction of the current injected into the outer edge channel is absorbed by the grounded central Ohmic contact (detected at $D$ ). Specific measurements of the tunneling between copropagating channels are discussed in the Appendix (Sec. V).

With inner channel loops formed [Fig. 2(c)], highamplitude oscillations of maximum visibility $\mathcal{V} \approx 80 \%$ and $40 \%$ are observed for the $L \simeq 24 \mu \mathrm{m}$ and $0.1 \mathrm{~mm}$ MZIs, respectively. Their sinusoidal shape is, however, perturbed by jumps as well as amplitude modulations, which are attributed to fluctuators such as moving charges in the MZI vicinity. A sudden variation in surrounding charges will indeed appear as a phase jump. In contrast, relatively rapid fluctuations with respect to the experimental integration time (approximately $1 \mathrm{~s}$ ) but slow with respect to the electron quantum coherence time will artificially reduce the amplitude of MZI oscillations, below their intrinsic value limited by $L_{\phi}$ according to Eq. (1). As illustrated with the emblematic single-electron transistor, individual charge fluctuators are usually influenced by surrounding gate voltages. Accordingly, we observe modulations of the phase jump density and of the amplitude of oscillations with gate voltages. Note that two sources of moving charges are specific to the present MZI implementation with inner channel loops: (i) the voltage bias applied to the very long surface gates used to form the loops and (ii) jumps in the number of electrons within each of the many inner channel loops (from the possible tunneling of electrons between outer channel and inner loops). We now further establish by a train of evidence that the large

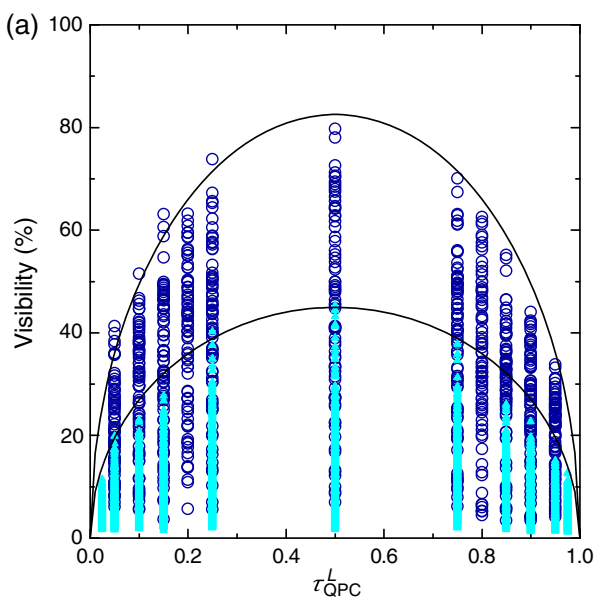

oscillations observed with inner channel loops result from the quantum interferences between the two MZI paths and that their maximum visibility accurately reflects $L_{\phi}$.

\section{OSCILLATION CHARACTERIZATION}

First, a well-defined plunger gate-voltage period of $2.2 \mathrm{mV}$ is observed for the smaller $L \simeq 24 \mu \mathrm{m}$ MZI, as directly evidenced from the power spectral density [dark blue lines in Figs. 2(d) and 2(f)]. A compatible but broader oscillation periodicity can also be perceived for the $L \simeq 0.1 \mathrm{~mm}$ MZI but only if the FFT analysis is restricted to plunger gate-voltage windows where the oscillation amplitude is relatively large [light blue line in Fig. 2(f)]. The period for $L \simeq 24 \mu \mathrm{m}$ with loops is shorter than without, as expected from the stronger influence of the plunger gate voltage. This reduction is a consequence of the quenched screening from isolated inner channel loops hosting a discrete number of electrons as compared to a copropagative inner channel. It also implies that any nearby moving charges will have a stronger impact on the MZI quantum phase.

Second, as shown in Fig. 3(a), the maximum oscillation visibility (highest symbols) follows the hallmark MZI signature $\sqrt{\tau_{\mathrm{QPC}}^{L}\left(1-\tau_{\mathrm{QPC}}^{L}\right)}$ (continuous lines) when varying the outer edge channel transmission probability across the left QPC beam splitter $\tau_{\mathrm{QPC}}^{L}$. For this purpose, we measure $\tau_{\mathrm{MZI}}\left(V_{\mathrm{pl}}\right)$ over many periods on both devices and for various settings of $\tau_{\mathrm{QPC}}^{L}$ at fixed $\tau_{\mathrm{QPC}}^{R} \simeq 0.5$ (see the Appendix). Each symbol in Fig. 3(a) (full and open

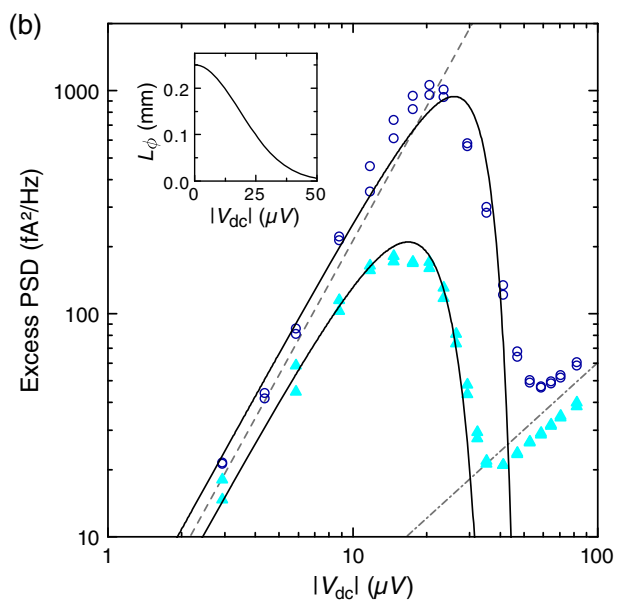

FIG. 3. Beam-splitter and bias-voltage tunings. Open (full) symbols are data points obtained on the $L \simeq 24(100) \mu \mathrm{m} \mathrm{MZI}$. (a) The local quantum oscillations' visibility in the presence of inner channel loops [Fig. 2(c)] separately extracted period per period along large $V_{\mathrm{pl}}$ sweeps is displayed as symbols versus the transmission probability $\tau_{\mathrm{QPC}}^{L}$ of the outer channel across the left QPC (at fixed $\tau_{\mathrm{QPC}}^{R} \simeq 0.5$ ). Continuous lines are Eq. (1)'s predictions for $L_{\phi}=0.25 \mathrm{~mm}$ with $L=24 \mu \mathrm{m}$ or $0.1 \mathrm{~mm}$. (b) The excess power spectral density of temporal fluctuations in the transmitted MZI current with respect to zero dc bias and averaged in $V_{\mathrm{pl}}$ is shown versus source $(S)$ dc voltage $V_{\mathrm{dc}}$. The gray straight lines represent a quadratic (dashed) and linear (dash-dotted) increase. The black continuous lines in the main panel display the noise contribution from phase fluctuations calculated with $L_{\phi}\left(V_{\mathrm{dc}}\right)=(0.25 \mathrm{~mm}) \times \exp \left[-\left(V_{\mathrm{dc}} / 26 \mu \mathrm{V}\right)^{2}\right]$ (shown in the inset). 
corresponding to the $L \simeq 24 \mu \mathrm{m}$ and $0.1 \mathrm{~mm}$ MZIs, respectively) displays the "locally" extracted visibility of the oscillations obtained by analyzing a restricted plunger gate-voltage window of one period $(2.2 \mathrm{mV})$. The close agreement between the highest data points and MZI expectations confirms that the observed oscillations result from the two-path quantum interferences.

Third, we find a quantitative data or theory agreement with the same $L_{\phi} \approx 0.25 \mathrm{~mm}$ for both devices, despite a factor of 4 in their size. The continuous lines in Fig. 3(a) are calculated using Eq. (1) with $L_{\phi}=0.25 \mathrm{~mm}$, the corresponding MZI length $L=24 \mu \mathrm{m}$ or $0.1 \mathrm{~mm}$, and $\tau_{\mathrm{QPC}}^{R}=0.5$. This agreement provides strong evidence that the measured maximum "local" visibility closely captures the intrinsic MZI visibility determined solely by $L_{\phi}$ (note that $L_{\phi}$ will otherwise be underestimated).

Fourth, as shown in Fig. 3(b), out-of-equilibrium measurements of the transmitted current noise around $0.86 \mathrm{MHz}$ further confirm the presence of MZI interferences accompanied by phase fluctuations and allow probing the energy dependence of $L_{\phi}$. The displayed data points represent measurements of the excess power spectral density of the current impinging on the electrode $D$ versus the dc bias voltage $V_{\mathrm{dc}}$ applied to the source electrode $S$. MZI phase variations, such as those produced by nearby charge fluctuators, are expected to manifest as a quadratic increase of the noise power at low $V_{\mathrm{dc}}$ (see the Appendix and Ref. [34]), as we experimentally observe. At larger bias, the generally expected reduction of $L_{\phi}$ also progressively diminishes the influence of the quantum phase and, consequently, the current noise induced by phase fluctuations. Experimentally, such a collapse is observed and can be accounted for using the same $L_{\phi}\left(V_{\mathrm{dc}}\right)$ for both devices: The two black continuous lines (main panel) are calculations based on Eq. (1) (see the Appendix, Eq. (A2)] using the empirically determined $L_{\phi}=(0.25 \mathrm{~mm}) \times$ $\exp \left[-\left(V_{\mathrm{dc}} / 26 \mu \mathrm{V}\right)^{2}\right]$ (shown in the inset). Ultimately, a linear noise increase is recovered as expected for the shotnoise contribution [34] (see the Appendix).

\section{DISCUSSION}

The large phase coherence length presently achieved provides information for the design of novel quantum Hall devices. It sets an upper bound to possibly relevant decoherence mechanisms along the quantum Hall edges in addition to the dominant interchannel coupling and narrows down the mechanisms for a frequently observed but still mysterious additional dissipation [25-27,35].

We establish that nearby metallic gates are completely compatible with large phase coherence lengths, despite the presence of many diffusive electrons. Note their beneficial screening of the long-range part of Coulomb interaction (to approximately $3.5 \mu \mathrm{m}$, the loop-gates' period, whether the loops are formed or not), which could otherwise provide an effective decoherence mechanism [36-39] as well as an unwanted coupling to spurious low-energy modes and distant channels $[35,40,41]$. In practice, a strong capacitive shortcut $(100 \mathrm{nF})$ is included at the low-temperature end of the electrical lines controlling the gates of our samples in order to further suppress both extrinsic and thermal noise sources.

We also find that the additional neutral modes predicted for a realistic smooth confinement potential at the edge [42-44] can essentially be ignored. Either these neutral modes are missing in the outer channel along our etcheddefined edges or they are very weakly coupled to the usual charge mode of the same channel. This finding is consistent with thermal conductance measurements across narrow constrictions perfectly transmitting one or several quantum Hall channels at integer bulk filling factors, where the extra heat transfer that would be expected from additional edge modes is not observed [45-47].

Finally, we mention that the two-dimensional quantum Hall bulk does not provide here a substantial path to quantum decoherence, at least when broken into small areas of a few micron squares (within the inner channel loops) and with the long-range part of Coulomb interaction screened by metallic gates. This finding contrasts with the observations of an unexpected heat flow away from the edge at lower filling factors [48-50] and of a long-distance capacitive coupling across the two-dimensional bulk [40,41].

\section{CONCLUSION}

We demonstrate that the electron quantum coherence in solid-state circuits can be extended to the macroscopic scale by strongly suppressing through circuit nanoengineering the dominant decoherence mechanism. The present implementation on quantum Hall edge channels is particularly well suited for the coherent control and long-distance entanglement of propagative electrons. Future optimizations include the understanding and suppression of the slow electron phase fluctuations here often, although not systematically, observed. Our work gives access to electron quantum optics devices of a higher complexity level, in line with the direction taken by this field of research $[1-3,18]$. More generally, increasing the electron phase coherence is essential to progress toward functional quantum devices involving multiple quantum manipulations, such as information processing with electronic flying qubits.

\section{ACKNOWLEDGMENTS}

This work is supported by the French RENATECH network, the national French program "Investissements d'Avenir" (Labex NanoSaclay, Grant No. ANR-10-LABX0035), and the French National Research Agency (project QuTherm, Grant No. ANR-16-CE30-0010-01).

E. S. and H. D. performed the experiment and analyzed the data with inputs from A. Aa., A. An., and F. P.; F. P. 
fabricated the sample with input from E. S. and H.D.; A. C., A. O., U. G. grew the 2DEG; F. P. led the project and wrote the manuscript with input from A. Aa., A. An., E. S., H. D. and U.G.; H.D. and E.S. contributed equally to this work.

\section{APPENDIX: EXPERIMENTAL METHODS}

\section{Samples}

Both samples are made of the same $\mathrm{Ga}(\mathrm{Al}) \mathrm{As}$ heterojunction hosting a two-dimensional electron gas of mobility $10^{6} \mathrm{~cm}^{2} \mathrm{~V}^{-1} \mathrm{~s}^{-1}$ and density $2.510^{11} \mathrm{~cm}^{-2}$ located $105 \mathrm{~nm}$ underneath the surface. They are nanofabricated by $e$-beam lithography, dry etching, and metallic deposition. The central metallic electrode (nickel [30 nm], gold [120 nm], and germanium [60 nm]) forms an Ohmic contact with the 2DEG obtained by thermal annealing (at $440^{\circ} \mathrm{C}$ for $50 \mathrm{~s}$ ) and is set to electrical ground through a suspended bridge. The two arms of each MZI are designed to be as symmetric as possible, such that the thermal smearing of the visibility induced by an asymmetry remains negligible by a large margin as previously observed $[17,33]$. The elongated shape of the central area is chosen to limit the overall magnetic flux enclosed between the two arms and, hence, the effect of environmental magnetic noise (e.g., from the pulse tube vibrations) on the particularly sensitive MZI phase in these very large devices. Note that a positive bias voltage of $+0.35 \mathrm{~V}$ is applied to all used gates during cooldown. This is a widespread procedure in $\mathrm{Ga}(\mathrm{Al}) \mathrm{As}$ devices to reduce the charge noise induced by biasing the gates, although it is probably not essential here due to the relatively low bias voltages used to form inner channel loops.

\section{Loop gate design}

Figure 4 recapitulates the different kinds of inner channel loops in the energy-relaxation experiment [31] [one inner loop enclosed only by the outer channel; see Fig. 4(a)] in the first MZI implementation [33] [inner loops enclosed by a metallic gate, the MZI outer channel, and another counterpropagating outer channel; see Fig. 4(b)] and in the present MZI implementation [inner loops enclosed by a metallic gate and the MZI outer channel; see Fig. 4(c)]. Now focusing on the present implementation, the gates'
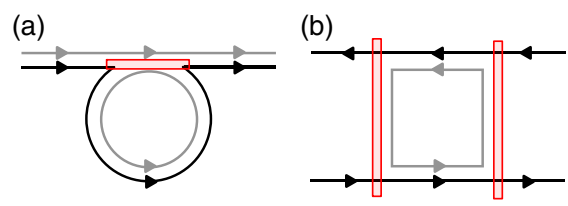

(c)

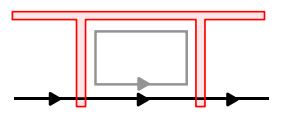

FIG. 4. Loop design. Inner loop design in previous energyrelaxation experiment [31] (a), previous MZI experiment [33], (b) and in the present implementation (c). The outer (inner) edge channel is represented by a black (gray) line. A schematic of the gates used to reflect the inner edge channel is displayed in red. width of $200 \mathrm{~nm}$ reflects a compromise between the separation with additional quantum Hall channels on the other side of the gates, which should be sufficiently large to result in a negligible coupling, and the wish to limit the $\nu=1$ area underneath the gates, as very weak interferences are often observed if the whole 2D bulk is set to $\nu=1$ (either by tuning $B$ without gates or using a broad top gate fully covering the 2D bulk; see, e.g., Ref. [24]). The distance between the inner channel loops and the propagative (inner) quantum Hall channel on the other side of the gates (opposite the MZI outer channel) should therefore be larger than $200 \mathrm{~nm}$. This is more than 1 order of magnitude larger than the narrow incompressible strip normally separating adjacent edge channels (typically $10 \mathrm{~nm}$ [51]). The loops' perimeter should also be chosen small enough such that the separation between the quantized electronic levels is larger than the available energy of approximately $k_{B} T$. Assuming a typical drift velocity between $10^{4}$ and $10^{5} \mathrm{~m} / \mathrm{s}$ along the sample edges, we find that the $9-\mu \mathrm{m}$ loop perimeter corresponds to a level spacing within 4.6 and $46 \mu \mathrm{eV}$, always larger than the thermal energy $\left(3 k_{B} T \simeq 2.6 \mu \mathrm{eV}\right.$ at $\left.10 \mathrm{mK}\right)$ and comparable to the characteristic $26-\mu \mathrm{V}$ dc bias voltage over which $L_{\phi}\left(V_{\mathrm{dc}}\right)$ is found to decrease [Fig. 3(b)]. Finally, the gates are designed elongated to minimize their overlap with the outer MZI edge channel, as at these locations their capacitive coupling is maximal and the lateral edge confinement is modified. Note also that one should be particularly careful about the electrical noise introduced by the measurement lines connected to the very long gates used to form the inner channel loops. These gates are indeed much more strongly coupled to the MZI phase than typical lateral plunger gates due to their very long size and because the inner loop efficiently mediates the capacitive coupling between the metallic gate and the MZI outer edge channel.

\section{Experimental setup}

The two simultaneously cooled devices are thermally anchored to the mixing chamber of a cryo-free dilution refrigerator. Electrical lines connected to the samples include multiple filters and thermalization stages. Note the important $R C$ filter $(200 \mathrm{k} \Omega, 100 \mathrm{nF})$ implemented at base temperature on the lines connected to the gates, including the long gates used to form the inner channel loops. Spurious high-frequency radiation is screened by two shields at base temperature. The fraction of transmitted current $\tau_{\mathrm{MZI}}$ is measured with lock-ins at a frequency below $200 \mathrm{~Hz}$ and using an effective integration time close to $1 \mathrm{~s}$ per point (corresponding to equivalent noise bandwidth of $0.8 \mathrm{~Hz}$ ). The power spectral density of temporal current fluctuations is measured over a much larger bandwidth of $180 \mathrm{kHz}$ around $0.86 \mathrm{MHz}$, using a homemade cryogenic amplifier and a tank circuit based on a superconducting coil. The temperature of electrons in the devices is extracted 
from the quantum shot noise across a quantum point contact (the right beam splitter QPC of the $L \simeq 24 \mu \mathrm{m}$ MZI set to $\tau_{\mathrm{QPC}}^{R} \simeq 0.5$ ). See Ref. [52] for further details on the same experimental setup.

\section{Central Ohmic contacts characterization}

The quality of the grounded central Ohmic contact is characterized by the ratio of reflected to impinging current. Ideally, there should be no reflected current. In practice, if the impinging current is carried only by the outer edge channel (used for the interferometer), the reflected current is found to be negligible for both devices (below 1\%). If the impinging current is carried by both the inner and outer edge channels, we find a reflected current in the range of $11 \%-21 \%$ corresponding to a $22 \%-42 \%$ reflection of the inner edge channel from the central Ohmic contact of the $L \simeq 24 \mu \mathrm{m}$ paths' MZI, whereas for the $L \simeq 0.1 \mathrm{~mm}$ MZI, the reflected current remains essentially negligible (below $1 \%)$. Note that a good Ohmic contact with the outer channel is assumed in Eq. (1) (an imperfect contact will further limit the amplitude of MZI oscillations).

\section{Tunneling between inner and outer channels}

Tunneling of electrons between adjacent copropagating channels is usually negligible at filling factor $\nu=2$. However, the propagation distances in the present devices can be considerable. Following standard procedures [53], we determine the electron interchannel tunneling along the MZI arms between the two QPC beam splitters when the inner edge channel is not formed into small loops. Note that the tunneling of electrons in the presence of small inner channel loops is expected to be much smaller because of the electronic level quantization within the loops and because of the Coulomb blockade of tunneling into (nearly) isolated islands (although this tunneling cannot be measured because there is no dc current toward closed loops). The tunneling between copropagative inner and outer edge channels is obtained by applying a small bias selectively on one of the two channels and by measuring at the end of the path the current in the other channel. We find that the tunneling remains small for the $L \simeq 24 \mu \mathrm{m}$ MZI [between $2.5 \%$ and $5 \%$ (approximately $0 \%$ ) of the injected current is detected on the second channel after propagating along the lower (upper) MZI arm]. The tunneling is more important for the $L \simeq 0.1 \mathrm{~mm}$ MZI [between $30 \%$ and $48 \%$ (between $10 \%$ and $26 \%$ ) of the injected current is detected on the second channel after propagating along the lower (upper) MZI path].

\section{Cross-talk characterization}

Changing a gate voltage also slightly influences the other nearby gates. We take into account this small capacitive cross-talk correction on the beam-splitter quantum point contacts (of at most 6\% attained for the lateral plunger gate effect on the nearby left QPC).

\section{Formation of inner channel loops}

The comb-shaped gates of homogeneous width $(200 \mathrm{~nm})$ are polarized with a positive voltage of $+0.35 \mathrm{~V}$ during the cooldown from room temperature. A broad gate-voltage window is found to fully reflect the inner quantum Hall channel while completely letting through the outer channel (with a minimal common window from 0 to $0.13 \mathrm{~V}$ that applies simultaneously to each arm of both devices). Such a behavior is usually observed on similar 2DEGs, thanks to the large energy separation between the two lowest Landau levels at filling factor $\nu=2$. Note that the results corresponding to closed inner channel loops that we present in the manuscript are not specific to a precise gate-voltage setting (chosen within the minimal common window) but representative of the general behavior observed when the inner edge channel loops are completely closed while the outer edge channel is fully propagative.

\section{Visibility of conductance oscillations versus QPC transmission}

Here we provide more details on the procedure followed to extract the oscillations visibility data displayed in Fig. 3(a). We perform relatively large plunger gate-voltage sweeps of $50 \mathrm{mV}$ corresponding to approximately 21 periods (with a step of $50 \mu \mathrm{V}$ corresponding to $1 / 46$ of a period) and repeat several times the same sweep (twice for the $L \simeq 24 \mu \mathrm{m}$ MZI, 14 times for the more challenging $L \simeq 0.1 \mathrm{~mm}$ MZI). Each sweep is then decomposed into one-period intervals with half a period of overlap between consecutive intervals, and a local visibility of the oscillations in $\tau_{\mathrm{MZI}}$ is extracted from $\mathcal{V} \equiv\left(\tau_{\mathrm{MZI}}^{\max }-\tau_{\mathrm{MZI}}^{\min }\right) /$ $\left(\tau_{\mathrm{MZI}}^{\max }+\tau_{\mathrm{MZI}}^{\min }\right)$ in each of these intervals. The symbols in Fig. 3(a) display the many different values of $\mathcal{V}$ obtained by this procedure.

\section{Temporal noise spectral density}

Here we provide more details on the noise data and calculations displayed in Fig. 3(b). The data points represent the excess power spectral density of the current detected on electrode $D$ [see Fig. 1(a)], i.e., the total noise from which is subtracted the equilibrium noise offset at $V_{\mathrm{dc}}=0$ (that includes the contribution of the amplification chain). To make sure that the noise dependence in the MZI quantum phase is fully averaged out, the displayed data represent the average of many noise measurements equally distributed in a range of the plunger gate voltage corresponding to several periods (240 [40] values of $V_{\mathrm{pl}}$ distributed over approximately five [2] periods for the $L \simeq 24[100] \mu \mathrm{m} \mathrm{MZI}$ ). The displayed calculations (continuous lines) include only the contribution of "slow" fluctuations in the MZI quantum phase $\delta \phi(t)$ detected 
within a $180-\mathrm{kHz}$ window around $0.86 \mathrm{MHz}$ and not the quantum shot-noise contribution that we further discuss below. From the relationship $\tau_{\mathrm{MZI}}(t)=$ $0.5\{1+\mathcal{V} \sin [\langle\phi\rangle+\delta \phi(t)]\}$, it is straightforward to obtain that the resulting noise in the transmitted current is given by [34]

$$
\left\langle I_{\delta \phi}^{2}\right\rangle_{\propto} \frac{V_{\mathrm{dc}}^{2} e^{4}}{h^{2}} \mathcal{V}^{2}
$$

with $h$ the Planck constant and $e$ the elementary electron charge. At low $V_{\mathrm{dc}}$ bias (as long as the oscillation visibility $\mathcal{V}$ is not significantly reduced), one thus expects a quadratic increase. Using the relationship between visibility and phase coherence length given Eq. (1), this expression becomes

$$
\left\langle I_{\delta \phi}^{2}\right\rangle \propto \frac{V_{\mathrm{dc}}^{2} e^{4}}{h^{2}} \exp \left(\frac{-4 L}{L_{\phi}}\right) .
$$

The calculations displayed as black continuous lines are obtained from Eq. (A2) using for both devices the same empirical expression $L_{\phi}\left(V_{\mathrm{dc}}\right)=0.25 \mathrm{~mm} \times$ $\exp \left[-\left(V_{\mathrm{dc}} / 26 \mu \mathrm{V}\right)^{2}\right]$ (displayed in the inset) the corresponding MZI length $L=24 \mu \mathrm{m}$ or $0.1 \mathrm{~mm}$ and where the unknown prefactor (depending on the number and coupling strength of the phase noise sources) is considered here as a free parameter for each device. The smaller quantum shotnoise contribution [not included in Eq. (A2)] is linear in $V_{\mathrm{dc}}$ and does not rely on the presence of MZI quantum interferences. As expected, if the vanishing current noise results from a quantum decoherence by "fast" phase fluctuations [34] (compared to the electron quantum coherence), the amplitude of the linear noise is found strongly suppressed compared to the naive expectation $\left\langle I^{2}\right\rangle=2 e\left(V_{\mathrm{dc}} e^{2} / h\right)\left\langle\tau_{\mathrm{MZI}}\right\rangle\left(1-\left\langle\tau_{\mathrm{MZI}}\right\rangle\right)$ by a factor of $4(6)$ for the MZI of arm length $L \simeq 24(100) \mu \mathrm{m}$.

\section{Comparison of voltage-bias robustness with and without inner channel loops}

In the absence of inner channel loops, the negligible MZI phase noise does not allow us to probe $L_{\phi}\left(V_{\mathrm{dc}}\right)$ through the power spectral density of the transmitted current's temporal fluctuations. However, on the $L \simeq 24 \mu \mathrm{m}$ MZI where quantum oscillations are visible without loops, it is possible to determine, versus dc voltage bias, their visibility $\mathcal{V}_{\text {diff }}$ in the transmitted differential current $d I_{\mathrm{MZI}} / d V_{\mathrm{dc}}$. The "diff" subscript is introduced here to clearly distinguish between, on the one hand, this usually measured $\mathcal{V}_{\text {diff }}$ and, on the other hand, the visibility $\mathcal{V}$ of oscillations in the total transmitted current $I_{\mathrm{MZI}}$ that is probed through noise measurements [Fig. 3(b)]. These two quantities are simply connected by the relation [54]

$$
\mathcal{V}_{\text {diff }}=\left|\mathcal{V}+V_{\mathrm{dc}} \partial \mathcal{V} / \partial V_{\mathrm{dc}}\right|
$$

Measurements of $\mathcal{V}_{\text {diff }}\left(V_{\mathrm{dc}}\right)$ on the $L \simeq 24 \mu \mathrm{m}$ MZI without loops are shown in Fig. 5 as open red circles. We find that $\mathcal{V}_{\text {diff }}$ displays a single side lobe with a first minimum at $\left|V_{\mathrm{dc}}\right| \simeq 5 \mu \mathrm{V}$ and becomes negligible below our experimental resolution at $\left|V_{\mathrm{dc}}\right| \gtrsim 15 \mu \mathrm{V}$. The data can be reproduced by the simple single side-lobe expression derived in Ref. [54] assuming a Gaussian phase averaging (continuous line in Fig. 5):

$$
\mathcal{V}_{\text {diff }}^{\text {Gaussian }}=\mathcal{V}_{0}\left|1-\frac{V_{\mathrm{dc}}^{2}}{V_{0}^{2}}\right| \exp \left(-\frac{V_{\mathrm{dc}}^{2}}{2 V_{0}^{2}}\right)
$$

with $\mathcal{V}_{0}=0.06$ the zero bias visibility and $V_{0}=5 \mu \mathrm{V}$ the characteristic voltage scale also corresponding to the position of the intermediate minimum. In order to compare the robustness of MZI interferences with and without inner channel loops, we convert the noise data in Fig. 3(b) into the corresponding $\mathcal{V}_{\text {diff }}$. The resulting $\mathcal{V}_{\text {diff }}$ is displayed in Fig. 5 as open dark blue circles and full light blue triangles for, respectively, the $L \simeq 24$ and $100 \mu \mathrm{m}$ MZI with loops. This conversion first involves the determination of $\mathcal{V}$ from Eq. (A1) (using the measured noise spectral density from which the linear shot-noise contribution observed at large $V_{\mathrm{dc}}$ is subtracted). The unknown proportionality coefficient in Eq. (A1) is fixed by adjusting the visibility at low bias with its direct $V_{\mathrm{dc}} \approx 0$ measurement displayed in Fig. 3(a). The resulting $\mathcal{V}$ is then injected into Eq. (A3) to obtain $\mathcal{V}_{\text {diff }}$.

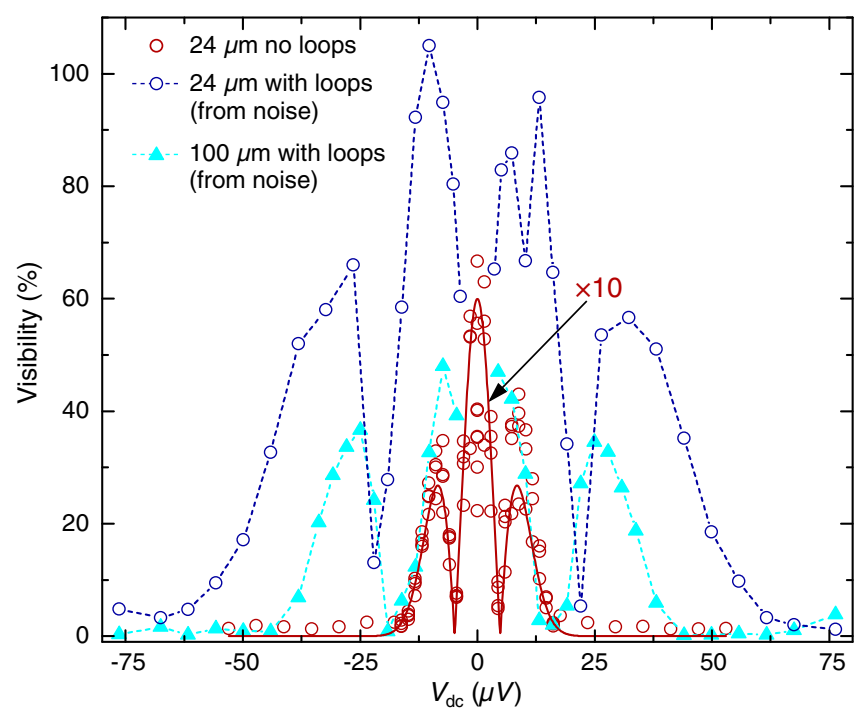

FIG. 5. Out-of-equilibrium visibility in the differential current $\mathcal{V}_{\text {diff }}$. The red circles represent measurements of the visibility of the oscillations in the differential transmitted current across the $L \simeq 24 \mu \mathrm{m}$ MZI without inner channel loops as a function of the applied dc bias voltage. The continuous red line is calculated from Eq. (A4) (see text). The dark blue circles (light blue full triangles) connected by dashed lines represent the differential visibility on the $L \simeq 24(100) \mu \mathrm{m}$ MZI with formed inner channel loops, which is extracted from the noise measurements displayed in Fig. 3(b) (see text). 
Comparing the two datasets at the same $L \simeq 24 \mu \mathrm{m}$ (open circles), we find that the robustness of the MZI visibility with $V_{\mathrm{dc}}$ is approximately 4 times larger in the presence of loops (dark blue) than without them (red).

[1] D. Glattli and P. Roulleau, Levitons for Electron Quantum Optics., Phys. Status Solidi B 254, 1600650 (2017).

[2] C. Bäuerle, D. C. Glattli, T. Meunier, F. Portier, P. Roche, P. Roulleau, S. Takada, and X. Waintal, Coherent Control of Single Electrons: A Review of Current Progress, Rep. Prog. Phys. 81, 056503 (2018).

[3] E. Bocquillon et al., Electron Quantum Optics in Ballistic Chiral Conductors, Ann. Phys. (Berlin) 526, 1 (2014).

[4] A. Bertoni, P. Bordone, R. Brunetti, C. Jacoboni, and S. Reggiani, Quantum Logic Gates Based on Coherent Electron Transport in Quantum Wires, Phys. Rev. Lett. 84, 5912 (2000).

[5] R. Ionicioiu, G. Amaratunga, and F. Udrea, Quantum Computation with Ballistic Electrons, Int. J Mod. Phys. B 15, 125 (2001).

[6] T. Stace, C. Barnes, and G. Milburn, Mesoscopic One-Way Channels for Quantum State Transfer via the Quantum Hall Effect, Phys. Rev. Lett. 93, 126804 (2004).

[7] T. Bautze, C. Sussmeier, S. Takada, C. Groth, T. Meunier, M. Yamamoto, S. Tarucha, X. Waintal, and C. Bauerle, Theoretical, Numerical, and Experimental Study of a Flying Qubit Electronic Interferometer, Phys. Rev. B 89, 125432 (2014).

[8] B. J. van Wees, L. P. Kouwenhoven, C. J. P. M. Harmans, J. G. Williamson, C. E. Timmering, M. E. I. Broekaart, C. T. Foxon, and J. J. Harris, Observation of Zero-Dimensional States in a One-Dimensional Electron Interferometer, Phys. Rev. Lett. 62, 2523 (1989).

[9] Y. Ji, Y. Chung, D. Sprinzak, M. Heiblum, D. Mahalu, and H. Shtrikman, An Electronic Mach-Zehnder Interferometer, Nature (London) 422, 415 (2003).

[10] I. Neder, N. Ofek, Y. Chung, M. Heiblum, D. Mahalu, and $\mathrm{V}$. Umansky, Interference between Two Indistinguishable Electrons from Independent Sources, Nature (London) 448 , 333 (2007).

[11] E. Bocquillon, V. Freulon, J.-M. Berroir, P. Degiovanni, B. Placais, A. Cavanna, Y. Jin, and G. Fève, Coherence and Indistinguishability of Single Electrons Emitted by Independent Sources, Science 339, 1054 (2013).

[12] Y. Nazarov and Y. Blanter, Quantum Transport (Cambridge University Press, Cambridge, England, 2009).

[13] H. Duprez et al., Transferring the Quantum State of Electrons across a Fermi Sea with Coulomb Interaction, arXiv:1902.07569.

[14] F. Pierre, A. B. Gougam, A. Anthore, H. Pothier, D. Esteve, and N. O. Birge, Dephasing of Electrons in Mesoscopic Metal Wires, Phys. Rev. B 68, 085413 (2003).

[15] Y. Niimi, Y. Baines, T. Capron, D. Mailly, F. Y. Lo, A. D. Wieck, T. Meunier, L. Saminadayar, and C. Bauerle, Quantum Coherence at Low Temperatures in Mesoscopic Systems: Effect of Disorder, Phys. Rev. B 81, 245306 (2010).
[16] F. Miao, S. Wijeratne, Y. Zhang, U. C. Coskun, W. Bao, and C. N. Lau, Phase-Coherent Transport in Graphene Quantum Billiards, Science 317, 1530 (2007).

[17] P. Roulleau, F. Portier, D. C. Glattli, P. Roche, A. Cavanna, G. Faini, U. Gennser, and D. Mailly, Direct Measurement of the Coherence Length of Edge States in the Integer Quantum Hall Regime, Phys. Rev. Lett. 100, 126802 (2008).

[18] M. Yamamoto, S. Takada, C. Bäuerle, K. Watanabe, A. D. Wieck, and S. Tarucha, Electrical Control of a Solid-State Flying Qubit, Nat. Nanotechnol. 7, 247 (2012).

[19] D. Wei, T. van der Sar, J. D. Sanchez-Yamagishi, K. Watanabe, T. Taniguchi, P. Jarillo-Herrero, B. I. Halperin, and A. Yacoby, Mach-Zehnder Interferometry Using Spinand Valley-Polarized Quantum Hall Edge States in Graphene, Sci. Adv. 3, e1700600 (2017).

[20] I. P. Levkivskyi and E. V. Sukhorukov, Dephasing in the Electronic Mach-Zehnder Interferometer at Filling Factor $\nu=2$, Phys. Rev. B 78, 045322 (2008).

[21] T. Giamarchi, Quantum Physics in One Dimension (Oxford University Press, New York, 2003).

[22] P. Roulleau, F. Portier, P. Roche, A. Cavanna, G. Faini, U. Gennser, and D. Mailly, Noise Dephasing in Edge States of the Integer Quantum Hall Regime, Phys. Rev. Lett. 101, 186803 (2008).

[23] L. V. Litvin, A. Helzel, H.-P. Tranitz, W. Wegscheider, and C. Strunk, Edge-Channel Interference Controlled by Landau Level Filling., Phys. Rev. B 78, 075303 (2008).

[24] I. Gurman, R. Sabo, M. Heiblum, V. Umansky, and D. Mahalu, Dephasing of an Electronic Two-Path Interferometer, Phys. Rev. B 93, 121412(R) (2016).

[25] H. le Sueur, C. Altimiras, U. Gennser, A. Cavanna, D. Mailly, and F. Pierre, Energy Relaxation in the Integer Quantum Hall Regime, Phys. Rev. Lett. 105, 056803 (2010).

[26] K. Itoh, R. Nakazawa, T. Ota, M. Hashisaka, K. Muraki, and T. Fujisawa, Signatures of a Nonthermal Metastable State in Copropagating Quantum Hall Edge Channels, Phys. Rev. Lett. 120, 197701 (2018).

[27] E. Bocquillon, V. Freulon, J.-M. Berroir, P. Degiovanni, B. Plaçais, A. Cavanna, Y. Jin, and G. Fève, Separation of Neutral and Charge Modes in One-Dimensional Chiral Edge Channels, Nat. Commun. 4, 1839 (2013).

[28] H. Inoue, A. Grivnin, N. Ofek, I. Neder, M. Heiblum, V. Umansky, and D. Mahalu, Charge Fractionalization in the Integer Quantum Hall Effect, Phys. Rev. Lett. 112, 166801 (2014).

[29] M. Hashisaka, N. Hiyama, T. Akiho, K. Muraki, and T. Fujisawa, Waveform Measurement of Charge- and SpinDensity Wavepackets in a Chiral Tomonaga-Luttinger Liquid, Nat. Phys. 13, 559 (2017).

[30] A. Marguerite, C. Cabart, C. Wahl, B. Roussel, V. Freulon, D. Ferraro, C. Grenier, J. M. Berroir, B. Placais, T. Jonckheere et al., Decoherence and Relaxation of a Single Electron in a One-Dimensional Conductor, Phys. Rev. B 94, 115311 (2016).

[31] C. Altimiras, H. le Sueur, U. Gennser, A. Cavanna, D. Mailly, and F. Pierre, Tuning Energy Relaxation along Quantum Hall Channels, Phys. Rev. Lett. 105, 226804 (2010). 
[32] C. Cabart, B. Roussel, G. Fève, and P. Degiovanni, Taming Electronic Decoherence in 1D Chiral Ballistic Quantum Conductors, Phys. Rev. B 98, 155302 (2018).

[33] P. A. Huynh, F. Portier, H. le Sueur, G. Faini, U. Gennser, D. Mailly, F. Pierre, W. Wegscheider, and P. Roche, Quantum Coherence Engineering in the Integer Quantum Hall Regime, Phys. Rev. Lett. 108, 256802 (2012).

[34] F. Marquardt and C. Bruder, Effects of Dephasing on Shot Noise in an Electronic Mach-Zehnder Interferometer, Phys. Rev. B 70, 125305 (2004).

[35] T. Krähenmann et al., Auger-Spectroscopy in Quantum Hall Edge Channels: A Possible Resolution to the Missing Energy Problem, arXiv:1902.10065.

[36] E. Sukhorukov and V. Cheianov, Resonant Dephasing in the Electronic Mach-Zehnder Interferometer, Phys. Rev. Lett. 99, 156801 (2007).

[37] J. Chalker, Y. Gefen, and M. Veillette, Decoherence and Interactions in an Electronic Mach-Zehnder Interferometer, Phys. Rev. B 76, 085320 (2007).

[38] D. Kovrizhin and J. Chalker, Multiparticle Interference in Electronic Mach-Zehnder Interferometers, Phys. Rev. B 81, 155318 (2010).

[39] M. Schneider, D. Bagrets, and A. Mirlin, Theory of the Nonequilibrium Electronic Mach-Zehnder Interferometer., Phys. Rev. B 84, 075401 (2011).

[40] M. Hashisaka, K. Washio, H. Kamata, K. Muraki, and T. Fujisawa, Distributed Electrochemical Capacitance Evidenced in High-Frequency Admittance Measurements on a Quantum Hall Device, Phys. Rev. B 85, 155424 (2012).

[41] N.-H. Tu, M. Hashisaka, T. Ota, Y. Sekine, K. Muraki, T. Fujisawa, and N. Kumada, Coupling between Quantum Hall Edge Channels on Opposite Sides of a Hall Bar, Solid State Commun. 283, 32 (2018).

[42] A. MacDonald, S. Yang, and M. Johnson, Quantum Dots in Strong Magnetic Fields: Stability Criteria for the Maximum Density Droplet, Aust. J. Phys. 46, 345 (1993).

[43] C. Chamon and X. Wen, Sharp and Smooth Boundaries of Quantum Hall Liquids, Phys. Rev. B 49, 8227 (1994).
[44] I. Aleiner and L. Glazman, Novel Edge Excitations of TwoDimensional Electron Liquid in a Magnetic Field, Phys. Rev. Lett. 72, 2935 (1994).

[45] S. Jezouin, F. D. Parmentier, A. Anthore, U. Gennser, A. Cavanna, Y. Jin, and F. Pierre, Quantum Limit of Heat Flow Across a Single Electronic Channel, Science 342, 601 (2013).

[46] M. Banerjee, M. Heiblum, A. Rosenblatt, Y. Oreg, D. E. Feldman, A. Stern, and V. Umansky, Observed Quantization of Anyonic Heat Flow, Nature (London) 545, 75 (2017).

[47] E. Sivre, A. Anthore, F. D. Parmentier, A. Cavanna, U. Gennser, A. Ouerghi, Y. Jin, and F. Pierre, Heat Coulomb Blockade of One Ballistic Channel, Nat. Phys. 14, 145 (2018).

[48] C. Altimiras, H. le Sueur, U. Gennser, A. Anthore, A. Cavanna, D. Mailly, and F. Pierre, Chargeless Heat Transport in the Fractional Quantum Hall Regime, Phys. Rev. Lett. 109, 026803 (2012).

[49] V. Venkatachalam, S. Hart, L. Pfeiffer, K. West, and A. Yacoby, Local Thermometry of Neutral Modes on the Quantum Hall Edge, Nat. Phys. 8, 676 (2012).

[50] H. Inoue, A. Grivnin, Y. Ronen, M. Heiblum, V. Umansky, and D. Mahalu, Proliferation of Neutral Modes in Fractional Quantum Hall States, Nat. Commun. 5, 4067 (2014).

[51] D. B. Chklovskii, B. I. Shklovskii, and L. I. Glazman, Electrostatics of Edge Channels, Phys. Rev. B 46, 4026 (1992).

[52] Z. Iftikhar, A. Anthore, S. Jezouin, F. D. Parmentier, Y. Jin, A. Cavanna, A. Ouerghi, U. Gennser, and F. Pierre, Primary Thermometry Triad at $6 \mathrm{mK}$ in Mesoscopic Circuits, Nat. Commun. 7, 12908 (2016).

[53] B. van Wees, E. M. M. Willems, C. J. P. M. Harmans, C. W. J Beenakker, H. van Houten, J. G. Williamson, C. T. Foxon, and J. J. Harris, Anomalous Integer Quantum Hall Effect in the Ballistic Regime with Quantum Point Contacts, Phys. Rev. Lett. 62, 1181 (1989).

[54] P. Roulleau, F. Portier, D. C. Glattli, P. Roche, A. Cavanna, G. Faini, U. Gennser, and D. Mailly, Finite Bias Visibility of the Electronic Mach-Zehnder Interferometer, Phys. Rev. B 76, 161309(R) (2007). 